\title{
Value-Based Healthcare and Enhanced Recovery After Surgery Implementation in a High-Volume Bariatric Center in Italy
}

\author{
Giulia Goretti $^{1}$ (D) Giuseppe M. Marinari ${ }^{2} \cdot$ Elena Vanni $^{3} \cdot$ Chiara Ferrari $^{4}$
}

Published online: 24 February 2020

(C) The Author(s) 2020

\begin{abstract}
Background Bariatric surgery is the most effective treatment for patients affected by morbid obesity. The Enhanced Recovery After Surgery (ERAS) protocol increases clinical outcomes, but the most recent literature shows incomplete patients' adherence. This study aims to demonstrate the feasibility of applying a Value-Based Healthcare (VBHC) strategy associated with ERAS to increase patients' engagement and outcomes.

Method A multiprofessional team redesigned the process considering ERAS recommendations and patients' feedbacks. Outcomes that matter to patients were defined with structured patients' interviews and collected in the electronic clinical record. Adherence to the pathway and the cost of the cycle of care were measured to demonstrate sustainability. A model was developed to grant its replicability.

Results A total of 2.122 patients were included. The lowest adherence to the protocol for a single item was $82 \%$. $74 \%$ of excess weight loss; $90 \%$ better comorbidities control; $77.5 \%$ had no pain after surgery; $61 \%$ no postoperative nausea and vomiting. Zero mortality; $1.8 \%$ overall morbidity; $0.4 \%$ readmission and reoperation rate within 30 days. The average length of stay is 2.1 days. Patient-Reported Outcome Measures (PROMs) documented increased productivity and quality of life.

Conclusion Building a caring relationship by a multidisciplinary team, adding patient wellness in a VBHC framework on top of ERAS as a patient-centered approach, increases patients' engagement and adherence to the pathway of care, resulting in better health outcomes (clinical and PROMs). The Value-Based Model is sustainable and replicable; it represents the prototype for redesigning other pathways and may become a model for other organizations.
\end{abstract}

Keywords Bariatric surgery $\cdot$ Value-based healthcare $\cdot$ Patient-centered care $\cdot$ Patient wellness $\cdot$ Performance measures $\cdot$ Quality improvement

\section{Introduction}

Morbid obesity is an increasingly prevalent condition worldwide. It is a chronic disease associated with long-term

Giulia Goretti

giulia.goretti@humanitas.it

1 Quality Department, Humanitas Clinical and Research Center IRCCS, via Manzoni, 5620089 Rozzano, MI, Italy

2 Bariatric Surgery, Humanitas Clinical and Research Center-IRCCS, via Manzoni, 5620089 Rozzano, MI, Italy

3 Performance audit, Humanitas Clinical and Research CenterIRCCS, via Manzoni, 5620089 Rozzano, MI, Italy

4 Anesthesia, Humanitas Clinical and Research Center-IRCCS, via Manzoni, 5620089 Rozzano, MI, Italy comorbidities (i.e., diabetes, cardiac and respiratory diseases, and malignant neoplasms), reduced overall survival (higher any cause-any age mortality), mental illness (i.e., depression and anxiety), and poorer quality of life [1].

Morbid obesity has become a serious international public health concern with a considerable impact on both direct (i.e., drugs) and indirect costs (productivity losses due to working days lost and reduced productivity at work) [2,3]. Indeed, the direct healthcare cost for obese adults is $42 \%$ higher than that for healthy adults [4]. Moreover, the estimated obesity-related medical cost in the USA is $\$ 147$ billion/year on the top of indirect costs, related to morbidity, mortality, and productivity loss [2]. For instance, job absenteeism alone costs approximately $\$ 4.3$ billion per year in the USA [5].

Bariatric surgery is currently the most effective treatment for morbid obesity, resulting in both sustained weight loss and improvement of obesity-related comorbidities [6]. An 
increasing number of bariatric procedures have been performed worldwide during the last 20 years and much effort has been made to identify efficient clinical management strategies for these patients.

The Enhanced Recovery After Surgery (ERAS) program is a clinical management approach designed to improve perioperative outcomes and decrease patient's length of hospital stay (LOS), leading to reduced healthcare costs [7, 8]. Worldwide recommendations for an ERAS approach to bariatric patients are still under implementation although preliminary positive results [9].

Clinical evidence [10-12] reported a substantial issue concerning patients' adherence to ERAS protocol. That is a relevant topic considering the relationship between adherence and survival [13] and outcome [12].

Value-Based Health Care (VBHC) is a strategic management framework that maximizes the ratio between health outcomes and costs [14-18]. Developed in 2006, it is based on three acting principles: (i) building value for the patients; (ii) basing the organization of medical practice on medical conditions and care cycles; (iii) measuring outcomes and economic costs [19]. To implement VBHC within the organizations, there is a 6-step approach [14] (Appendix 1). Case studies have been published, mainly in northern Europe, to describe the method and potential benefits in the application of the approach [19-25]. Up to this date, no studies have been performed in Italy and for obese patients worldwide to our knowledge.

Starting from ERAS, we redesigned the organizational pathway with the involvement of a multi-professional team, patients and their families. We defined and measured the patient-relevant outcomes and we compared them to the costs, as the strategy that VBHC suggests.

In this observational study, we show how to implement a VBHC strategy, starting from ERAS principles applied to a high volume bariatric center and the feasibility of this approach to achieve excellent clinical outcomes and better quality of life without increasing costs, through the involvement and engagement of patients.

The aim of this work is to increase patients' adherence to their own cycle of care, to show the feasibility of this approach and to develop a replicable model.

\section{Methods}

In July 2015 , the bariatric surgery unit started its activity at a private and highly specialized academic hospital in northern Italy with strong assets and costs management and culture of measurement [26].

The bariatric team was already used to work combining clinical excellence and efficiency and brought its previous experience in Fast Track protocol; thinking about patient value, though, required a wider perspective.

For this reason, a multi-professional team (surgeon, anesthesiologist, lean manager, asset manager, scrub, pain and ward nurse, nutritionist, and psychologist) was organized to compose an integrated practice unit (IPU). Patients were interviewed by clinicians to collect their experiences and suggestions to improve their pathway of care. From July to October 2015 the team reached a consensus on the ERAS elements reported in Table 1. From November to December 2015, the team designed the process, and according to Lean principles (Appendix 2), the IPU defined the value for patients and clinicians.

Patients' value was defined as (i) excess weight loss; (ii) better control of comorbidities; (iii) quality of life improvement; and (iv) positive experience throughout the process.

From the clinicians' perspective value was represented by optimization of clinical parameters to get patients more fit for surgery and engaged about their own care which results in the reduction of hospital LOS and readmission rate [27, 28]. Patients' engagement became a strategic issue to be addressed to increase the adherence to the clinical protocol and, as a consequence, to improve outcomes.

\section{Intervention}

The IPU designed the value stream mapping (Appendix 3) both for the clinical and organizational processes (Table 2). The first contact with patients was through a dedicated reference person that gave them all the information required to be prepared for the first multidisciplinary visit. A multidisciplinary team followed patients during the whole pathway. Both individual meetings and group discussions were performed. In group meetings, structured as counseling, patients and their families shared experiences, questions, fears, and concerns with all bariatric IPU members. Patients already treated were also invited to share their bariatric surgery experience. In the preadmission phase, blood tests and clinical-instrumental examination were performed according to evidence from recent literature [29]. As an example, chest X-rays were not routinely executed, but only when a clinical condition was present. During surgery, a mini-invasive surgical approach was adopted. Early mobilization and fluid intake (30 min after surgery) granted lower pain, nausea, and vomiting.

Every patient was asked to write a diary with all the activities and symptoms experienced (drinking, walking, respiratory rehabilitation, pain, vomiting) with the aim of proactively self-monitoring the recovery progression. The nurses checked and registered all the data.

Direct contact with clinicians was guaranteed by a $24 / 7$ unique phone number answered by one of the team members, a structured follow-up phone call was held by a case 
Table 1 ERAS recommendations, reviewed by our clinicians for (a) preoperative, (b) intraoperative, and (c) postoperative care in bariatric surgery

Recommendation Action

(a)

Perioperative information, education, counseling

Prehabilitation and exercise

Preoperative fasting

(b)

Perioperative fluid management

Standardized anesthetic protocol

PONV (postoperative nausea/vomiting)

Surgical approach

(c)

Early postoperative nutrition

Early postoperative mobilization
Patients and caregivers receive preoperative counseling

During the month preceding surgery patients are recommended to walk and do respiratory exercises. Any coexistent disease should be compensated and patients with CPAP ventilation for OSA (Obstructive Sleep Apnea) should be compliant to therapy for at least 4 weeks before anesthesia.

Fluid intake up to $2 \mathrm{~h}$ and solid food up to $4 \mathrm{~h}$ prior to anesthesia induction.

Goal-directed fluid therapy during surgery, the start of oral fluid intake 30 min after surgery, intravenous support only if low compliance or clinical direction

Dexmedetomidine infusion for premedication

Short-acting anesthetic agents (i.e., desforane, rocuronium)

Full reversal of neuromuscular blockade (sugammadex)

Opioid sparing analgesia

Structured approach to airway management

Bispectral Index (BIS) monitoring of anesthetic depth

Monitoring of neuromuscular blockade (TOF)

A multimodal approach to PONV prophylaxis is adopted in all patients

Laparoscopy

No drains or tubes

Start fluid intake 30 min after surgery; quantitative reporting of total daily intake in the clinical record

Start walking in the recovery room 30 min after surgery; quantitative reporting of total daily steps in the clinical record manager the week after discharge to early detect potential complications.

All data coming from these calls were collected in the electronic clinical records to allow reporting.

In January 2016, the first obese patient entered the new protocol, and we started measuring health performance. Thus, the intervention involved the following 2.122 patients until May 2018. One year after discharge patients were investigated by clinicians about their quality of life. Patients included in the one-year follow-up had surgery until June 2017.

\section{Outcomes}

Medical data were collected in the electronic clinical records, daily updated and accessible by every professional involved

Table 2 The bariatric standard process (in italics, the main changes introduced by the new process)

\begin{tabular}{|c|c|c|c|c|c|c|}
\hline Pre-assessment & Assessment & Preadmission & Surgery & $\begin{array}{l}\text { Recovery ( } 30 \mathrm{~min} \\
\text { after awakening) }\end{array}$ & Ward & Follow-up \\
\hline $\begin{array}{l}\text { Dedicated contact } \\
\text { center }\end{array}$ & $\begin{array}{l}\text { Multidisciplinary } \\
\text { (surgeon, } \\
\text { nutritionist, } \\
\text { psychologist) }\end{array}$ & In a single day & $\begin{array}{c}\text { Standardized } \\
\text { anesthesia } \\
\text { protocol }\end{array}$ & $\begin{array}{l}\text { Early } \\
\quad \text { mobilization }\end{array}$ & $\begin{array}{l}\text { A diary for } \\
\text { self-reporting } \\
\text { activities }\end{array}$ & Diary update \\
\hline \multirow[t]{3}{*}{$\begin{array}{l}\text { Checklist of questions } \\
\text { and exams to be } \\
\text { prepared for the } \\
\text { assessment }\end{array}$} & \multirow[t]{3}{*}{$\begin{array}{l}\text { Anesthesiology } \\
\text { checklist to early } \\
\text { detection of } \\
\text { critical conditions }\end{array}$} & $\begin{array}{l}\text { Multidisciplinary } \\
\text { visit (surgeon, } \\
\text { anesthesiologist, } \\
\text { pain nurse) }\end{array}$ & $\begin{array}{l}\text { Dedicated team of } \\
\text { anesthesiologists }\end{array}$ & Early fluid intake & $\begin{array}{l}\text { Mobilization } \\
\text { and fluid } \\
\text { intake }\end{array}$ & \multirow[t]{3}{*}{$\begin{array}{l}\text { Standardized case } \\
\text { manager follow } \\
\text { up (1 week after } \\
\text { surgery) }\end{array}$} \\
\hline & & $\begin{array}{l}\text { Supplementary } \\
\text { exams only if } \\
\text { indicated after } \\
\text { the visit }\end{array}$ & $\begin{array}{l}\text { Mini-invasive } \\
\text { surgical } \\
\text { approach }\end{array}$ & $\begin{array}{l}\text { Respiratory } \\
\text { rehabilitation }\end{array}$ & $\begin{array}{l}\text { Respiratory } \\
\text { rehabilitation }\end{array}$ & \\
\hline & & $\begin{array}{l}\text { Counseling for } \\
\text { patients and } \\
\text { caregivers }\end{array}$ & $\begin{array}{l}\text { SMED and } 5 S \text { of } \\
\text { the operating } \\
\text { room }\end{array}$ & & $\begin{array}{l}\text { Pain nurse daily } \\
\text { check }\end{array}$ & \\
\hline
\end{tabular}


using any personal computer of the hospital. A set of key performance indicators (KPIs) was developed to measure improvement:

Value in Patients' Perspective Value in Patients' Perspective means excess weight loss (EWL) and better control of comorbidities. The percentage of EWL (\%EWL) is the primary clinical outcome after bariatric surgery, leading to better control of comorbidities and return to daily activities. It could be evaluated at any time after surgery. We reported \%EWL at shortterm (1 year) and medium-term follow-up ( 3 years) to measure the sustained weight loss. The main comorbidities were as follows: hypertension, obstructive sleep apnea syndrome (OSAS), and type 2 diabetes. Bariatric surgeons and nutritionists record \%EWL and recovery from comorbidities during the follow-up evaluations.

Quality-of-Life Improvement Quality-of-life improvement is quantified by Patient-Reported Outcome Measures (PROMs), chosen considering the quality of life for obese patients, using the Bariatric Analysis and Reporting Outcome System (BAROS) [30]: possibility or amount of physical activities, work capability, dressing, and sexual activity were collected 1 year after surgery.

Bariatric surgeons collect PROMs during the follow-up visit 1 year after discharge.

Process adherence Current literature [10-12] reported an average patients' adherence to ERAS items at $70 \%$, ranging from $60 \%$ to more than $80 \%$, depending on patients' age and the clinical phase. A relevant issue is to increase the adherence to protocol until a complete observance, considering its effect on survival [13] and outcomes [12]. We investigated the effect of patients' engagement and commitment on adherence with a consistent data collection. We analyzed the percentage of patients attending: (i) activities for motivation and preparation (counseling, prehabilitation, and personal diary's drawing up); (ii) ERAS protocol, including early mobilization and fluid intake in the postoperative phase to reduce pain and clinical complications; (iii) follow-up phone call and visit 1 year after surgery to grant the sustainability of the achieved results.

LOS was measured as a primary output of process' adherence.

Clinical Outcomes Clinical outcomes are as follows: mortality, morbidity, readmission and reoperation rates within 30 days after surgery; mortality 1 year after discharge; postoperative pain, nausea and vomiting in day zero, one and two after surgery and 1 week later. Access to our local data warehouse allowed direct comparison of data sets for each unit (i.e., bariatric surgery unit) with regional and national registers (i.e., AGENAS, National Agency for Regional Health Services).
Resources, Value, and Costs Cost control department measured the direct resources relate to the cost for the clinical treatment and the freed-up resources due to process optimization in bariatric surgery, available for other patients in the hospital. All the analyses related to resource consumption were registered in our data warehouse and used daily.

\section{Patient Involvement in the Research Project}

Patients and their families partnered with us during the whole process. At the beginning patients undergoing standard care were asked for feedbacks and suggestions to improve, using structured interviews.

During the implementation of the intervention, patients actively participated in their own care and worked on items with their care-givers.

To grant results' sustainability, periodic meetings with previous patients or patients to be and their caregivers were organized to keep them motivated, sharing their experience and having new proposals to improve.

This original contribution was a single-center observational cohort study of elective bariatric surgical patients. It aimed to implement the VBHC approach on ERAS protocol and to measure the impact of the engagement of patients and a multiprofessional team on outcomes, quality of life and costs, to prove its feasibility and playable.

\section{Results}

\section{Studied Patients}

From January 2016 to May 2018, we studied 2.122 morbidly obese patients (MOP), of whom $89 \%$ underwent sleeve gastrectomy and $11 \%$ gastric bypass surgery.

The demographic characteristics of the patients are shown in Table 3.

Table 3 Demographics of the studied patients

\begin{tabular}{lc}
\hline Male & $30 \%$ \\
Female & $70 \%$ \\
Age (years) & $42 \pm 11$ \\
BMI & $45 \pm 6.6$ \\
Hypertension & $65 \%$ \\
Obstructive sleep apnea syndrome & $15 \%$ \\
Type 2 diabetes & $7.8 \%$ \\
\hline
\end{tabular}




\section{Outcome}

\section{Value for Patients}

Percentage Excess Weight Loss The \%EWL measured at the short-term follow-up after surgery was $74.05 \%$, consistently higher than the European average $(58.49 \%)$ registered in the database of the European Accreditation Council for Bariatric Surgery (EAC-BS).

The medium-term follow-up data, 3 years after surgery, further improved the obtained results. The $\% \mathrm{EWL}$ was $82.03 \%$, steadily higher than the European average $(51.89 \%)$ (source: EAC-BS).

Recovery from Comorbidities Together with \%EWL, improvement of other clinical conditions and comorbidities was investigated 1 year after surgery and compared with those of other European countries. Considerable results were obtained, as shown in Fig. 1. Patients, for the most part, recovered from comorbidities, thus, discontinuing or reducing pharmacological treatment. The most consistent result was on type 2 diabetes: $81 \%$ of patients discontinued the therapy, obtaining normal values of glycathed hemoglobin (HbAlc).

Quality-of-Life Improvement as PROMs One year after surgery patients underwent an interview assessing quality-of-life parameters. Seventy-seven percent of the patients reported to work better and more than before the procedure. Eighty-nine percent were able to practice physical activities, and 52\% reported a longer training time. Ninety-two percent of patients were buying clothes everywhere and not only in special shops for oversized customers. Ninety percent graded their sexual life as "good" and $48 \%$ reported an improvement.

Process Adherence ERAS recommendations (Table 1) were applied and compliance was monitored by physicians using a pre-operative questionnaire. All the patients attended counseling, prehabilitation, and exercises. Regarding preoperative tests, the process optimization resulted in waste reduction and patients spent $40 \%$ less time at the hospital completing all the exams in a single morning.

Ninety-two percent of them had oral fluid intake up to 2 $8 \mathrm{~h}$ before surgery. Preoperative fluid management and PONV prophylaxis were adopted in $100 \%$ of patients. Regarding anesthesia, a standardized anesthetic protocol was fully applied. All the surgical procedures were performed using laparoscopy and in $98 \%$ of the cases, no drains or tubes were placed.

Postoperative intensive care admittance was almost zero $(0.2 \%)$, mainly because of tight clinical management of all the comorbidities before surgery (i.e., CPAP for OSAS, glycemic control, hypertension control).

All patients received support to start oral fluid intake and mobilization $30 \mathrm{~min}$ after surgery. Moreover, $98 \%$ of them recorded their progress in a diary.

This standardized patient-centered approach resulted in an average LOS, of 2.1 days for all the 2.122 patients. Responders of follow-up phone calls from the case manager were $82 \%, 7$ days after surgery and compliance to the 1-year follow-up visit with the surgeon was $83.4 \%$.

Clinical Outcomes Retrospective data analysis from the studied population revealed zero mortality and $1.8 \%$ overall morbidity within 30 days of surgery. Mortality was zero 1 year after discharge. Both readmission and reoperation rates were $0.4 \%$ within 30 days of surgery.

During the whole hospitalization, $77.5 \%$ of MOP experienced no pain at all (NRS $<3$ ). $22.5 \%$ of the patients reported pain or discomfort and were successfully treated with rescue therapy as protocol. Regarding Post Operative Nausea and Vomiting (PONV), 28\% reported mild nausea, 11\% vomiting, $61 \%$ reported no symptoms. One week after discharge the prevalence of nausea and vomiting was $6 \%$.

Costs Table 4 summarizes the clinical changes responsible for the main impact on resources, the additional costs and freed-up resources. While the new process entails additional direct resources for counseling for every patient, it frees up time on chest X-ray machine
Fig 1 MOP comorbidities: comorbidities resolved (no drugs any more), improved (reducing drugs' number or dose), or without change after 12 months for our patients compared with those of European data

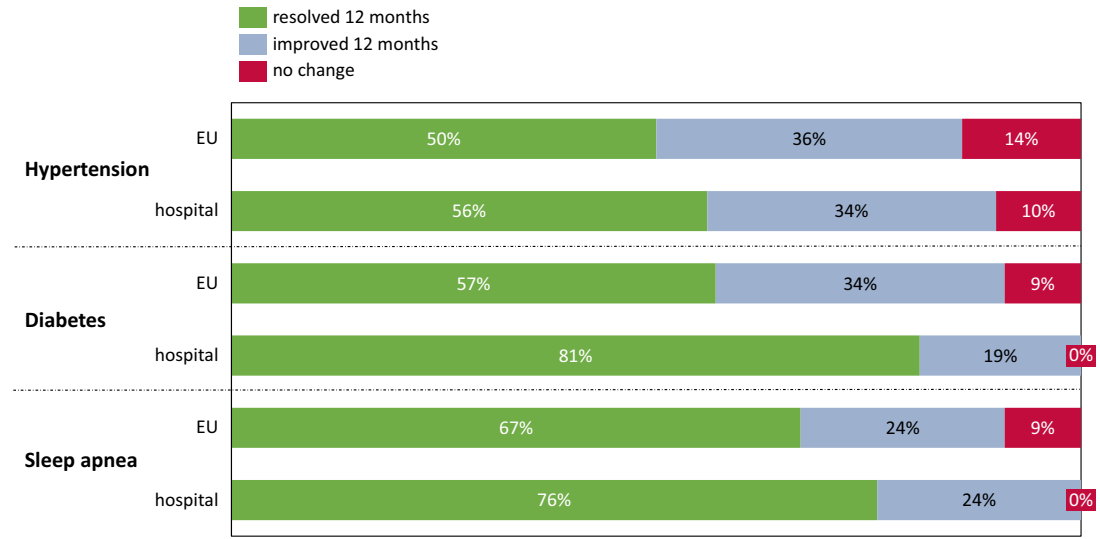


Table 4 Main clinical changes that impact on resources

\begin{tabular}{|c|c|c|c|c|}
\hline & Previous protocol & New intervention & $\begin{array}{l}\text { Additional } \\
\text { costs }(*) \text { in } €\end{array}$ & $\begin{array}{l}\text { Freed-up } \\
\text { resources }(*) \text { in } €\end{array}$ \\
\hline Counseling and prehabilitation & None & All patients & 100 & \\
\hline Chest X-ray & All patients & Only if needed due to specific clinical condition & & -53.3 \\
\hline ERAS recommendations & Intraoperative applied & fully applied & & -9.9 \\
\hline \multirow[t]{2}{*}{ Postoperative intensive care unit usage } & Scheduled for some patients & None scheduled & & -38.5 \\
\hline & & & 100 & -102 \\
\hline
\end{tabular}

*Average for patient

which could offer additional chest X-rays to other patients. Because of the new anesthetic protocol and the early post-surgical mobilization (30 min after surgery), patients had better pain and vomiting control, leading in turn to $40 \%$ lower drug prescriptions.

Given the financial value of resources involved, additional costs associated with counseling are compensated by the additional revenues obtained from the freed-up chest X-rays, no ICU utilization, and lower drug usage.

\section{Discussion}

Our intervention confirms the positive impact of bariatric surgery on clinical outcomes and significant improvement of quality of life for MOP. The care of these patients requires much more than an effort on clinical and technical skills only. Understanding how patients and their families' motivation, education, and involvement can impact their outcomes has been the key to implement the cultural change needed to achieve better overall results. We redesigned the bariatric process taking into deep consideration all the milestones mentioned above starting from an evidence-based clinical pathway (according to ERAS principles [9] shaped on MOP needs as shown in Table 1 and Nice Guidelines [29]) and international benchmarks [31-33], to put the basis of a standardized model of care integrated with a structured motivational and educational support.

The turning point to reach this goal has been redesigning the process of adopting a multidisciplinary approach and considering the different points of view of entities involved: (i) patients and families; (ii) clinicians; and (iii) cost and asset management and control.

Translating clinical and patients' needs in organizational changes has been possible placing patient value first.

From a methodological point of view, we created a framework to maximize the value coming from a multidisciplinary inclusive approach and we invested in patients' engagement to obtain full adherence (from $82 \%$ to $100 \%$ ), leading to better clinical and patient-oriented outcomes. Also, we measured costs of the full cycle of care as the VBHC approach suggests (Appendix 1).
Engaging patients and caregivers, we experimented full compliance with key clinical steps: all patients were able to drink and walk 30 min after surgery in the recovery room and we recorded less request of analgesic and antiemetic drugs, likely as a direct consequence of the early movement. Nurses accompany patients on their first excursions and, progressively, as they begin to show confidence and a positive attitude, they can walk with their familiars or friends along with the ward. Self-reporting activities on a diary keep patients motivated and aware of their progresses, which contributes significantly to compliance. Consistent evidence already supported the positive impact on outcomes from patients and caregivers motivation, education, and direct involvement in their own pathway of care $[34,35]$.

Twenty percentage points of patients' adherence higher than previous studies confirmed the relevance to add to a patient-centered care approach (ERAS) a patient wellness framework (VBHC on top of ERAS). The core is the care relationship between the patient and the multidisciplinary team.

Other research studies showed the positive impact of VBHC on quality and costs, even in complex surgical systems [24] but without exploring the important impact on the quality of life for patients.

For the first time, PROMs have been measured together with clinical outcomes in our hospital. Even if PROMs are currently being debated, there is a global consensus on their relevance with traditional measures of healthcare to improve clinical care [36-38]. Consistent evidence suggests obese employees could only reach approximately $80 \%$ of the productivity of a normal-weight worker [2, 39]. One year after surgery, $77 \%$ of our patients reported to work better and more than before the intervention. The above-stated issue was strictly related to better performance in physical activities and a positive approach to daily life.

In our opinion, based on the overall results (clinical, PROMs, and adherence), patients' and caregivers' involvement with the IPU personnel since the beginning, through counseling, multidisciplinary approach, and close follow-up, have been the key elements of success and the real innovation in our organization. 
We measured the economic impact of the new pathway and it is sustainable because every economic saving (i.e., no ICU utilization and lower drug usage) is invested in quality and activities to involve patients (i.e., counseling).

Communication among IPU members, patients, and board management staff has been crucial during this process.

Dedicated meetings were organized to share results and progress.

To our knowledge, this is the first VBHC study implemented in Italy. We are convinced that this could be a good example of patients' and families' engagement in since they ultimately became part of the team.

The measurement of impact on social costs is the next challenge and a further step for an even larger improvement.

The Value-Based Model developed from bariatric surgery experience has become the prototype for redesigning other surgical pathways in our hospital (breast cancer, esophagus, and prostate cancer), and the aim is to be a case study for other organizations to set this approach.

We studied and implemented the new pathway for all MOP, and its limit is that it is not a randomized controlled trial. Having worked with already elaborated results from databases of clinical records, we recognize as limitation of our study the lack of a detailed analytic approach based on single-patient data.

\section{Conclusion}

This methodological and descriptive report shows the feasibility of applying Value-Based Healthcare and ERAS to obtain patients' engagement in the full cycle of care.

Building a caring relationship by a multidisciplinary team, adding patient wellness in a VBHC framework on top of ERAS as a patient-centered approach, increases patients' adherence to the pathway of care, resulting in better health outcomes (clinical and PROMs).

It is sustainable: all the savings were invested in activities for patients' motivation.

It is replicable: other surgical pathways in our hospital have been redesigned using the bariatric model.

Acknowledgments The authors thank all the members of the bariatric team: surgeons, anesthesiologists, scrubs, ward and recovery nurses, the nutritionist, the case manager, and the secretary. We are grateful to Dr. Patrizia Meroni for her support in spreading this experience and the Board of our hospital that believed in our vision. We thank the staff, operations management team, statistical office, medical direction, and management for the support in the implementation phase.
Contributors GG, GMM, and CF conceptualized and designed the study. EV contributed to writing the costs analysis.

\section{Compliance with Ethical Standards}

Conflict of Interest The authors declare that they have no conflict of interest.

Human and Animal Rights and Informed Consent All procedures performed in studies involving human participants were in accordance with the ethical standards of the institutional and/or national research committee and with the 1964 Helsinki declaration and its later amendments or comparable ethical standards.

Informed consent was obtained from all individual participants included in the study.

\section{Appendix 1. Value-Based Healthcare}

It has been developed by M. Porter and E. Teisberg [14] to delivering superior patient value, measuring value as patient health outcomes per dollar spent.

It is based on 6 simple steps:

1. Organize care into integrated practice units (IPUs) around patient medical conditions

2. Measure outcomes and costs for every patient

3. Move to bundled payments for care cycles

4. Integrate care delivery systems

5. Expand geographic reach

6. Build an enabling information technology platform

In VBHC, the multiprofessional team is crucial, including patients and their families with an approach "patient center, doctor driven", as well as international collaboration, costeffectiveness within the full cycle of care and the development of models having potential for upscaling.

In VBHC, it is essential to incrementally improve initiatives and scale-up to attract enough patient volume.

\section{Appendix 2. Lean Principles}

The term Lean was coined by J.P. Womack and D.T. Jones to describe the Toyota Production System that managed to get by the half of space, labor effort, capital, inventory, and far fewer than half the defect and safety incidents [40]. Lean is more than a toolset; it is a management system for continuous improvement and for employee engagement that allows to create problem solvers [41].

It is based on 5 principles [42]:

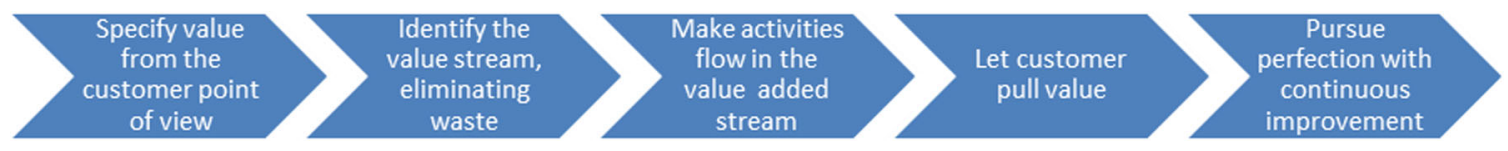


Even if lean was born in the automotive industry, every type of organization, including healthcare, has to face customer and employee satisfaction, cash flow, and quality.

Lean methodology moved from manufactory to healthcare in 2001, with Virginia Mason Institute, Seattle Children's Hospital, and Royal Bolton Hospitals as pioneers. Our hospi- tal started the lean journey in 2011, and we are close to the 5 principle interpretation for healthcare suggested by Intermountain Healthcare [43], inspired by Spear and Bowen [44].

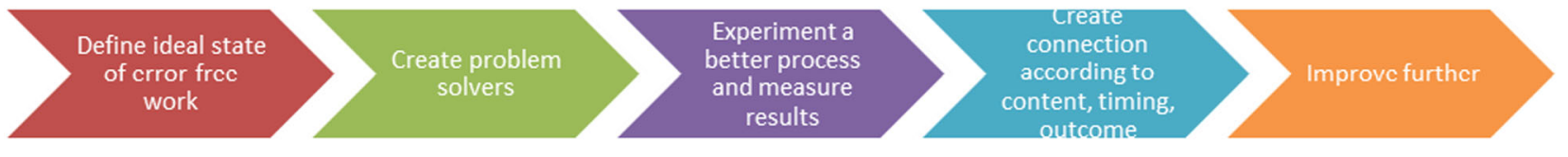

1. Every change must move the organization closer to this ideal, meaning:

- Exactly what the patient needs, defect-free

- One by one, customized to each patient

- On demand, exactly as requested

- Immediate response to problems or changes

- No waste

- Safe for patients, staff, and clinicians: physically, emotionally, and professionally

2. Problems are quickly identified because not conform to the ideal. Employees are the experts and the organization must help them to become problem solvers, in the recognition that employees are assets rather than expensive items.

3. The process must change as soon as a better way is known. Countermeasures are implemented as scientific experiments and results are measured.

4. Processes improve when connections are direct, simple, and binary-doing so makes them prompt, efficient, and reliable.

5. These simple rules not only define the ideal work process but enable workers to quickly see opportunities to improve.

\section{Appendix 3. Value Stream Map [43]}

VSMs visually show the key people, material, and information flows required to deliver a product or service. It is used to understand the high-level view of a process to distinguish value-added and nonvalue-added activities. As an example in healthcare, a value-added activity is the doctor's interview to obtain clinical information, whereas nonvalue-added activity is the waiting time.

Once a problem is recognized, specific problem solving can be focused to improve the process using lean tools.
As a consequence, a "future state map" is created that outlines a clear goal toward which the team applies its improvement efforts.

Open Access This article is licensed under a Creative Commons Attribution 4.0 International License, which permits use, sharing, adaptation, distribution and reproduction in any medium or format, as long as you give appropriate credit to the original author(s) and the source, provide a link to the Creative Commons licence, and indicate if changes were made. The images or other third party material in this article are included in the article's Creative Commons licence, unless indicated otherwise in a credit line to the material. If material is not included in the article's Creative Commons licence and your intended use is not permitted by statutory regulation or exceeds the permitted use, you will need to obtain permission directly from the copyright holder. To view a copy of this licence, visit http://creativecommons.org/licenses/by/4.0/.

\section{References}

1. Nguyen NT, Varela JE. Bariatric surgery for obesity and metabolic disorders: state of the art. Nat Rev Gastroenterol Hepatol. 2017;14: $160-9$.

2. Goettler A, Grosse A, Sonntag D. Productivity loss due to overweight and obesity: a systematic review of indirect costs. BMJ Open. 2017;7:e014632.

3. Raine KD, Nykiforuk CI, Vu-Nguyen K, et al. Understanding key influencers' attitudes and beliefs about healthy public policy change for obesity prevention. Obesity. 2014;22:2426-33.

4. Finkelstein EA, Trogdon JG, Cohen JW, et al. Annual medical spending attributable to obesity: payer-and service-specific estimates. Health Aff. 2009;28(5):w822-31.

5. Cawley J, Rizzo JA, Haas K. Occupation-specific absenteeism costs associated with obesity and morbid obesity. J Occup Environ Med. 2007;49(12):1317-24.

6. Kissler HJ, Settmacher U. Bariatric surgery to treat obesity. Semin Nephrol. 2013;33(1):75-89.

7. Ljungqvist $\mathrm{O}$, Scott M, Fearon KC. Enhance recovery after surgery. A review. JAMA Surgery. 2017;152(3):292-7.

8. Singh PM, Panwar R, Borle A, et al. Efficiency and safety effects of applying ERAS protocols to bariatric surgery: a systematic review with meta-analysis and trial sequential analysis of evidence. Obes Surg. 2017;27(2):489-501. 
9. Thorell A, MacCormick AD, Awad S, et al. Guidelines for perioperative care in bariatric surgery: enhanced recovery after surgery (ERAS) society recommendations. World J Surg. 2016;40(9): 2065-83.

10. Pędziwiatr M, Mavrikis J, Witowsk J, et al. Current status of enhanced recovery after surgery (ERAS) protocol in gastrointestinal surgery. Med Oncol. 2018;35(6):95.

11. Wolk S, Distler M, Müssle B, et al. Adherence to ERAS elements in major visceral surgery. An observational pilot study. Langenbeck's Arch Surg. 2016;401(3):349-56.

12. Gianotti L, Fumagalli RU, De Pascale S, et al. Association between compliance to an enhanced recovery protocol and outcome after elective surgery for gastric cancer. Results from a Western population-based prospective multicenter study. World J Surg. 2019;43(10):2490-8.

13. Gustafsson UO, Oppelstrup H, Thorell A, et al. Adherence to the ERAS protocol is associated with 5-year survival after colorectal cancer surgery: a retrospective cohort study. World J Surg. 2016;40(7):1741-7.

14. Porter ME, Teisberg EO. Redefining health care: creating valuebased competition on results. Harvard Business Review Press 2006.

15. Porter ME, Teisberg EO. How physicians can change the future of health care. JAMA. 2007;297:1103-11.

16. Porter ME, Teisberg EO. Redefining competition in health care. Harv Bus Rev. 2004;82:64-76.

17. Porter M, Lee TH. The strategy that will fix health care. Harv Bus Rev. 2013;91:50-705.

18. Kaplan RS, Porter ME. How to solve the cost crisis in health care. Harv Bus Rev. 2011;89:46-52.

19. Nilsson K, Bååthe F, Andersson AE, et al. Experiences from implementing value-based healthcare at a Swedish University Hospital - a longitudinal interview study. BMC Health Serv Res. 2017;17:169.

20. Wilson H, Gole J, Bharat M, et al. Value based healthcare. Advanc Manag. 2016;9:1-8.

21. Bozic KJ, Wright JG. Value-based healthcare and orthopaedic surgery: editorial comment. Clin Orthop Relat Res. 2012;470:1004-5.

22. Sallin K, Makdessi L, Sjogreen J. The Swedish Society of Medicine: value-based health care is loaded with problems, but also opportunities. Lakartidningen. 2015;112:C96L.

23. Wohlin J, Aspelin P, Rehnqvist N, et al. Value-based health care shifts the focus from care production to health. Lakartidningen. 2015;112:C96H.

24. Kirkpatrick JR, Marks S, Slane M, et al. Using value-based analysis to influence outcomes in complex surgical systems. J Am Coll Surg. 2015;220:461-8.

25. McLaughlin N, Buxey F, Chaw K, et al. Value-based neurosurgery: the example of microvascular decompression surgery. J Neurol Surg. 2014;120:462-72.

26. Bohmer RMJ, Pisano GP, Tang N. Istituto Clinico Humanitas (A). Harvard Business School Case 2002;603-063.

27. Cakir H, van Stijn MF, Lopes Cardozo AM, et al. Adherence to enhanced recovery after surgery and length of stay after colonic resection. Color Dis. 2013;15(8):1019-25.
28. Gillis C, Gill M, Marlett N, et al. Patients as partners in enhanced recovery after surgery: a qualitative patient-led study. BMJ Open. 2017:7(6).

29. National Institute for Health and Care Excellence: Clinical guidelines. London: National Institute for Health and Care Excellence (UK); 2003. https://www.nice.org.uk/guidance/ng45. Accessed April 2016.

30. Oria HE, Moorehead MK. Updated bariatric analysis and reporting outcome system (BAROS). Surg Obes Relat Dis. 2009;5(1):60-6.

31. Jacobsen HJ, Bergland A, Raeder J, et al. High-volume bariatric surgery in a single center: safety, quality, cost-efficacy and teaching aspects in 2,000 consecutive cases. Obese Surgery. 2012;22(1): $158-66$.

32. Ronellenfitsch U, Schwarzbach M, Kring A, et al. The effect of clinical pathways for bariatric surgery on perioperative quality of care. Obes Surg. 2012;22(5):732-9.

33. Bergland A, Gislason H, Raeder J. Fast-track surgery for bariatric laparoscopic gastric bypass with focus on anaesthesia and perioperative care. Experience with 500 cases. Acta Anaesthesiol Scand. 2008;52(10):1394-9.

34. Cook D, Thompson JE, Dearani JA, et al. How Mayo Clinic is using iPads to empower patients. Harv Bus Rev. 2014;

35. Gillis C, Gill M, Marlett N, et al. Patients as partners in enhanced recovery after surgery: a qualitative patient-led study. BMJ Open. 2017;7(6): 017002.

36. Abola RE, Bennett-Guerreo E, Kent M, et al. American Society for Enhance Recovery and Perioperative Quality Initiative Joint Consensus Statement on patient-reported outcomes in a enhance recovery pathway. Anesth Analg. 2018;126(6):1874-81.

37. Fleisher LA, Ko CY. Enhance recovery after surgery: is it time to drive patient-reported outcome through robust measurement? Anesth Analg. 2018;126(6):1801-2.

38. Waljee JF, Ghaferi A, Cassidy R, et al. Are patient-reported outcomes correlated with clinical outcomes after surgery? A population-based study. Ann Surg. 2016;264(4):682-9.

39. Tsai SP, Ahmed FS, Wendt JK, et al. The impact of obesity on illness absence and productivity in an industrial population of petrochemical workers. AEP. 2008;18(1):8-14.

40. Womack JP, Jones DT, Roos D. The machine that changed the world: the story of lean production. Free Press. 1990;

41. Graban M. Lean hospitals: improving quality, patient safety, and employee engagement. CRC Press. 2012:1-18.

42. Marchwinski C, Shoot J. Lean Lexicon: a graphical glossary for lean thinkers: Lean Enterprise Institute; 2003.

43. Jimmerson C, Weber D, Sobek D. Reducing waste and errors: piloting lean principles at Intermountain Healthcare. Jt Comm J Qual Patient Saf. 2005;31(5):249-57.

44. Spear S, Bowen HK. Decoding the DNA of the Toyota production system. Harv Bus Rev. 1999;76:97-106.

Publisher's Note Springer Nature remains neutral with regard to jurisdictional claims in published maps and institutional affiliations. 